\title{
347.
}

\section{ON THE NOTION AND BOUNDARIES OF ALGEBRA.}

[From the Quarterly Journal of Pure and Applied Mathematics, vol. vi. (1864), pp. 382-384.]

I Do not admit the assertion, that the idea of number is derived from that of time, it appears to me that it is derived from that of succession in time or space indifferently. But I would rather say that the idea of cardinal number is derived and abstracted from that of ordinal number, viz. (distinguishing the expressions 'set' and 'series,' the latter being used to designate a set of things considered as arranged in a definite order), if we have a series of things $a, b, c, d$, \&c., or say a series of words first, second, third, fourth, \&c.; then any set of things $X, N, Y, P, Q$, \&c., taking them up one after the other, no matter in what order, and coordinating them with the terms of the series $a, b, c, d$, \&c. or with the words, first, second, third, fourth, \&c.the last of them will be coordinated with a definite term of the series $a, b, c, d$, \&c., or with a definite term of the series first, second, third, fourth, \&c.; that is, the set, whatever be the assumed order of the terms, or (what is the same thing) without assuming any order therein, will have a certain property; viz. in the set $X, N, Y, P, Q$, where the last term is coordinated with $e$ or with the word fiftl, the property is that the set consists of five things: and so in general the set consists of a certain (cardinal) number of things, such cardinal number being the number corresponding to the rank in the series $a, b, c, d$, \&c., of the term wherewith is coordinated the last term of the set, or corresponding with the like ordinal number in the series first, second, third, fourth, \&c.

The foregoing remarks are made to some extent incidentally, but they have a bearing on the distinction in kind which exists e.g. between the proposition $1+1+1+1=4$, and the proposition which for ordinary purposes would be expressed as $1+1+$ \&c. 
( $n$ terms $)=n$, but which is better expressed in the form $1_{1}+1_{2}+\ldots+1_{n}=n$, where the subscript numbers merely distinguish between the different unities which are added altogether.

I use the term Algebra in a wide sense as including, or indeed I might say identical with, Finite Analysis, and excluding Infinite Analysis; but in speaking of it as identical with Finite Analysis I include in that term part of what might be considered Infinite Analysis; viz. many of the theorems relating to infinite series or other successions of operations, e.g.

$$
(1-x)\left(1+x+x^{2}+\ldots \text { ad inf. }\right)=1,
$$

really belong to Finite Analysis; for what is asserted is that the coefficient of the term of indefinite rank, say $x^{n}$, is a finite series equal in value to zero (this coefficient in fact is $1-1$ which is $=0$ ). On the other hand the theorem

$$
1-\frac{x^{2}}{1.2}+\frac{x^{4}}{1.2 .3 .4}-\& c .=\left(1-\frac{x^{2}}{\pi^{2}}\right)\left(1-\frac{x^{2}}{4 \pi^{2}}\right) \ldots
$$

the truth whereof depends on the equations

$$
\frac{\pi^{2}}{2}=\frac{1}{1^{2}}+\frac{1}{2^{2}}+\frac{1}{3^{3}}+\ldots \text { ad inf., \&c., \&c., }
$$

which are not arithmetically verifiable, belongs strictly to Infinite Analysis.

Algebra is an Art and a Science; quì Art, it defines and prescribes operations which are either tactical or else logistical; viz. a tactical operation is one relating to the arrangement in any manner of a set of things; a logistical operation (I prefer to use the new expression instead of arithmetical) is the actual performance, so as to obtain for the result a number, of any arithmetical operations (of course, given operations) finite in number, since these alone can be actually performed, upon given numbers. And quà Science Algebra affirms à priori, or predicts, the result of any such tactical or logistical (or tactical and logistical) operations. An equation such as $1+4+10=15$ is not an algebraical theorem; it is merely the assertion that the sum of the numbers $1,4,10$ is that number, viz. 15, which is the sum of the numbers in question. And, similarly, the equation $1+1+1=3$ is not an algebraical theorem. But on the other hand, the equation $1+1+1+\ldots(n$ terms $)=n$, is an algebraical theorem; in the equivalent form $1_{1}+1_{2}+\ldots+1_{n}=n$, (where $1_{k}=1$ ) it is not different in kind from the equation $1+2+3 \ldots+n=\frac{1}{2} n(n+1)$, or say $1_{1}+1_{2}+\ldots+1_{n}=\frac{1}{2} n(n+1)$, (where $1_{k}=k$ ) which is certainly an algebraical theorem. And this leads to the remark, that every algebraical theorem rests ultimately on a tactical foundation. In fact, whether we prove the last-mentioned theorem in the easiest way by writing

$$
\begin{aligned}
& 1+2+3 \ldots+n=S \\
& n+(n-1)+(n-2) \ldots+1=S
\end{aligned}
$$


and therefore $2 S=(n+1)+(n+1)+\ldots(n$ terms $)=n(n+1)$ or $S=\frac{1}{2} n(n+1)$; or by induction by showing that the theorem, if true for $n$, is true for $(n+1)$, (this depends on the equation $\frac{1}{2} n(n+1)+(n+1)=(n+1)\left(\frac{1}{2} n+1\right)=\frac{1}{2}(n+2)$; the proof is equally a tactical one; it is always tactic which determines what logistical operations are to be performed.

Although it may not be possible absolutely to separate the tactical and logistical operations; for in (at all events) a series of logistical operations, there is always something that is tactical, and in many tactical operations (e.g. in the Partition of Numbers) there is something which is logistical, yet the two great divisions of Algebra are Tactic and Logistic. Or if, as might be done, we separate Tactic off altogether from Algebra, making it a distinct branch of Mathematical Science, then (assuming in Algebra a knowledge of all the Tactic which is required) Algebra will be nothing else than Logistic. 